\title{
The International Conference on the Freeze-Drying of Pharmaceuticals and Biologicals
}

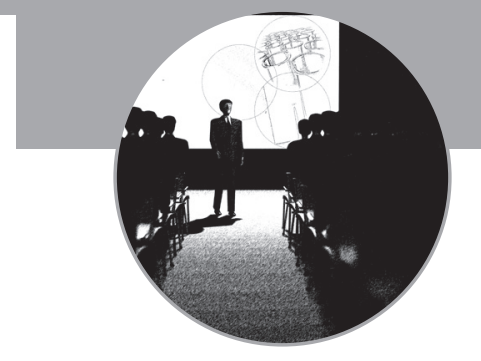

\section{The International Conference on the Freeze-Drying of Pharmaceuticals and Biologicals Garmisch-Partenkirchen, Germany, 28 September-I October, 2010}

The stability of pharmaceuticals and biological products is a major challenge for the industry. While some preparations have adequate stability as a liquid formulation, many biological molecules must be dried in order to achieve shelflives compatible with a commercial product. In addition, the technology for conducting freeze-drying and sample analysis is continually improving. Researchers from all relevant areas discussed critical aspects and new developments at the conference of Freeze-Drying of Pharmaceuticals and Biologicals.

Pharmaceuticals require a sufficient shelf-life in order to be developed as commercial products. The more recent interest in biotechnology-related products (e.g., proteins, DNA and RNA) has created a renewed interest in the freeze-drying process and the stability of biological molecules in the dried state. This topic draws upon a wide range of disciplines including materials sciences, physics, engineering, biology and chemistry. This conference occurs every other year and rotates between GarmischPartenkirchen, Germany and Breckenridge, $\mathrm{CO}$, USA. The organizations responsible for presenting this conference are the University of Munich (Ludwig Maximilians University, Germany), University of Connecticut (CT, USA) and the Dane O. Kildsig Center for Pharmaceutical Processing Research (IN, USA). The latest developments in both formulation advancements and technology improvements are presented at this conference, which first convened in 1998. The presentations are divided into three broad areas: materials sciences, biotech formulations and technical operations. In addition to these three symposia, there is time dedicated to poster presentations and a Soap Box session that allows exhibitors to present their latest products and technology.

In order to prepare the nonspecialist for the detailed topics discussed in the symposia, a short course by Mike Pikal (University of Connecticut) and Steve Nail (Baxter Pharmaceuticals, IN, USA) was offered the day before the conference. Pikal and Nail are two of the most distinguished mentors in the science of freeze-drying, and their short course introduced scientists to the basics of physics, process engineering and formulation as they relate to lyophilization. In addition, these two experts enlivened the short course with their personal anecdotes of more than 60 years of practical experience in both academia and industry.

The conference began with an introductory lecture from Ed Trappler (Lyophilization Technology, PA, USA) describing the history of freeze-drying dating back to the 1930s, including a large focus on blood plasma products, antibiotics and vaccines. He discussed the 'explosion' of interest in freeze-drying since the 1980s predominantly due to new antibiotics and antiviral compounds. More recently, there has been renewed interest in lyophilization due to the development of biotechnology products, including new vaccine technology, oncolytics and biopharmaceuticals (including monoclonal antibodies, live viruses and siRNA).

The first symposium on materials sciences discussed topics associated with the physical states of dried systems, and Marc Descamps (University of Lille, France) described the processing and characterization of crystalline and amorphous materials. Since most biotechnology products require an amorphous phase for optimal stability, Mike Pikal and Marc Cicerone (National Institute of Standards and Technology, MD, USA) gave presentations focused on the mobility within glassy matrices and how dynamics within the glass affect protein stability. These presentations discussed the consequences of polymorphism, and Raj Suryanarayanan (University of Minnesota, MN, USA) presented data demonstrating dramatic $\mathrm{pH}$ swings that can occur during the freeze-drying process due to buffer crystallization. These $\mathrm{pH}$ swings can be dramatic (several $\mathrm{pH}$ units), and may result in
Thomas J Anchordoquy

School of Pharmacy, 12700 E 19th Ave, Aurora, CO 80045, USA

Tel.: + I 3037246113

Fax: + I 3037247266

E-mail: tom.anchordoquy

@ucdenver.edu 
a much more severe destabilization of the active pharmaceutical ingredient than would be predicted by the $\mathrm{pH}$ determined before and after freezing. In addition, speakers demonstrated new data on sugars that are typically used in freeze-dried products (e.g., trehalose) showing that crystallization and degradation of the sugar can occur during storage. Furthermore, Tom Anchordoquy (University of Colorado, CO, USA) presented evidence that the drying step of a freeze-drying process generates reactive oxygen species that can foster chemical degradation. Robin Bogner (University of Connecticut) also presented a talk on approaches to measure variability within a batch and how tolerance affects design space. At the end of each session, time was allotted for a panel discussion with all speakers from the session. While some questions were taken immediately after each presentation (time permitting), this panel discussion allows questions to be asked of all the speakers. The preeminent sage of freeze-drying, Alan MacKenzie, was in charge of these sessions and contributed his keen insight and authoritative comments while coordinating the discussion.

The second symposium on Biotechnology formulations began with presentations on particulate formulations, including vaccines and nanosuspensions. Pierre Chouvenc (SanofiPasteur, Lyon, France) described the use of some atypical exipients (e.g., xanthane and urea) in the development of an inactivated polio virus vaccine, and Henning Gieseler (University of Erlangen, Germany) discussed the rationale for using poloxamers to sterically stabilize drug nanosuspensions during freeze-drying. The potential of freeze-drying to foster the adsorption of buffer species to vaccine particles, which ultimately reduces electrostatic stabilization, was presented by Ted Randolph (University of Colorado), and the consequences of cake collapse were brought into question by Kathrin Schersch (Novartis, Basel, Switzerland). Typically, collapse of the dried cake is considered detrimental because it reduces the physical appearance ('pharmaceutical elegance'), and is typically thought to compromise stability as well as reconstitution times. However, data were presented that challenged this dogma, and raised questions regarding the unsuitability of collapsed cakes considering the potential advantages of drying at higher temperatures. The last speaker in the symposium, Liz Topp (Purdue University, IN, USA), presented data on thiol/disulfide interchange reactions in dried, amorphous solids, highlighting the differences of these chemical reactions in the dried state as compared with solutions. This session concluded with a lively panel discussion including all the speakers. After lunch, a poster session and Exhibitors Soap Box session rounded out the second day of the conference.

The final symposium focusing on technical operations began with presentations on modeling the freeze-drying process. Alina Alexeenko (Purdue University) described the simulation of vapor/ice dynamics, and Julien Andrieu (University of Lyon, France) and Daniel Gunderud (IMA Edwards, NY, USA) discussed advantages of utilizing organic solvents to speed evaporation. The next three presentations described improved methods for monitoring samples during the lyophilization process. Steve Nail discussed the use of tunable diode laser absorption spectroscopy in cycle development and optimization in production scale equipment. Michael Wiggenhorn (Coriolis, Martinsried, Germany) introduced novel optical fiber sensors that allow for the sensitive detection of some unexplained thermal events in addition to conventional thermal signals used in process development. Thomas De Beer (University of Gent, Belgium) described the in-line monitoring of samples with multiple spectroscopic techniques (near infrared, Raman and plasma emission) that may provide a more intimate look at changes that occur to both the active molecule and the excipient matrix during the freeze-drying process.

To close out the conference, Stephanie Passot (AgroParisTech, Thierval-Grignon, France) detailed the challenges associated with freezedrying microorganisms and the impact of parameter variability on product quality. Evgenyi Shalaev (Pfizer, CT, USA) discussed the impact of residual moisture content on mobility within the glassy matrix, polarity, hydrolysis rates and local $\mathrm{pH}$. Ultimately, all of these factors can play a role in protein stability in a freeze-dried cake, and the data presented make it clear that a drier product does not always exhibit enhanced stability. The final presentation involved the control of nucleation during the freezing step of freezedrying; a vexing problem that leads to sample variability with regard to drying characteristics and product quality. Robert Sever (Praxair Inc., IL, USA) described a newly developed technology whereby the lyophilization chamber is pressurized and then de-pressurized to promote ice nucleation within the sample vials. Although the precise mechanism by which this occurs is not 


\section{CONFERENCE Report | News \& ANalysis}

fully understood, convincing evidence demonstrating the utility of this approach garnered considerable interest from the attendees.

After a dynamic panel discussion including questions for all the speakers in the final symposium, some concluding remarks from Professors Gerhard Winter and Wolfgang Friess (University of Munich, Germany) brought a close to the conference. The next conference on the Freeze-Drying of Pharmaceuticals and Biologicals is planned for the summer of 2012 in Breckenridge, CO, USA. Anyone interested in getting on the mailing list for this conference should contact Elizabeth Anderson at the University of Connecticut School of Pharmacy: Liz.Anderson@UConn.edu.

\section{Acknowledgement}

This conference was dedicated to Professor Louis Rey who could not be present this year. The attendees sent their good wishes for Professor Rey in the hope that he will be able to participate in the next conference.

\section{Financial \& competing interests disclosure}

This work was supported by NIH/NIBIB under grant RO1 EB006398-01A1. The author has no other relevant affiliations or financial involvement with any organization or entity with a financial interest in or financial conflict with the subject matter or materials discussed in the manuscript apart from those disclosed.

No writing assistance was utilized in the production of this manuscript. 